\title{
Osmocapillary phase separation
}

\section{Citation}

Liu, Qihan, and Zhigang Suo. 2016. Osmocapillary Phase Separation. Extreme Mechanics Letters 7: 27-33. doi:10.1016/j.eml.2016.02.001.

\section{Published Version}

doi:10.1016/j.eml.2016.02.001

\section{Permanent link}

http://nrs.harvard.edu/urn-3:HUL.InstRepos:30367423

\section{Terms of Use}

This article was downloaded from Harvard University's DASH repository, and is made available under the terms and conditions applicable to Open Access Policy Articles, as set forth at http:// nrs.harvard.edu/urn-3:HUL.InstRepos:dash.current.terms-of-use\#OAP

\section{Share Your Story}

The Harvard community has made this article openly available.

Please share how this access benefits you. Submit a story.

Accessibility 


\title{
Osmocapillary phase separation
}

Qihan Liu, Zhigang Suo

Harvard John A. Paulson School of Engineering and Applied Sciences, Kavli Institute for Bionano Science and Technology, Harvard University, MA 02138

\begin{abstract}
:
A covalent network of polymer can imbibe a solvent to form a gel. In a cavity on the surface of the gel, capillary force may suck the solvent out of the gel to form a pure liquid phase. We show that such osmocapillary phase separation occurs when capillarity balances osmosis, and when the diffusion of solvent in the gel prevails over the condensation of solvent from the vapor. Osmocapillary phase separation can occur even when the gel is isolated from the vapor, or when the solvent is nonvolatile. We relate osmocapillary phase separation to phenomena of practical significance, including the wettability of gels, the transparency of gels, the liquid bridge at the tip of an atomic force microscope, the adhesion between a gel and another substance, the surface morphology of gels, and the production of tight oil.
\end{abstract}

\section{Introduction}

Osmotic phenomena have appeared in writings since antiquity $[1,2]$. For example, to detach a block of desired size from a large rock, Egyptians would drive wedges of dry wood into slots in the rock, and then soak the wood with water till the swelling wood split the rock. As a second example, an animal bladder, filled with wine and plunged in water, swells greatly and sometimes even bursts. As a third example, the Bible reported that Moses turned brackish water into drinking water, possibly using an ion-exchanging process.

We now know the basic ingredients to set up osmosis. In a system of several substances, one substance-designated as solvent-can migrate from place A to place $\mathrm{B}$, but the other substances cannot. The chemical potential of the solvent in place A is higher than that in place B; the difference in the chemical potential provides the driving force for the migration of the 
solvent. The animal bladder is a semipermeable membrane: water can pass through it, but alcohol cannot. Outside the bladder, the pure liquid water has a high chemical potential of water. Inside the bladder, the wine is a mixture of alcohol and water, and has a low chemical potential of water. This difference in chemical potential of water motivates pure water outside the bladder to pass through the membrane and dilute the wine.

Osmosis plays essential roles in nature and engineering. Living cells undergo osmotic swelling and collapse when the aqueous solution outside the gel changes concentration. Tall trees demonstrate the spectacular power of osmosis [3]. When air is less humid than soil, the difference in humidity drives the ascent of sap in trees. In the xylem of tall trees, liquid water is often under hydrostatic tensile stress in excess of $1 \mathrm{MPa}$ [4]. Osmosis-induced high tensile stress in liquid water has been demonstrated in artificial trees on chips [5]. Biological and bioinspired actuators rely on swelling polymers in response to diverse stimuli [6, 7]. Superabsorbent diapers keep babies dry even when babies sit on swollen diapers [8]. Seals made of swelling elastomers are deployed in oilfields to enable hydraulic fracture [9]. Desalination of water has developed into an industry since the time of Moses [10].

In an osmotic phenomenon, osmosis-the tendency to mix the solvent to other substances-usually competes against some other forces. For the bladder filled with wine and plunged in water, osmosis competes against the elasticity of the membrane. During desalination, external forces must be applied to saltwater to push pure water through membranes, against osmosis. Desalination separates water from solutes, and is a process of reverse osmosis. During the ascent of sap, the trees harness the difference in the chemical potentials of water in the soil and in the air, separate water from the soil, and raise water to the treetops.

Here we explore a phenomenon in which osmosis competes against capillarity. We begin with a hydrogel-a covalent network of hydrophilic polymer swollen with water. The chemical potential of water in the hydrogel is readily set by equilibrating the hydrogel with a water vapor of known relative humidity, or with an aqueous solution of known concentration [11]. Assume 
that the hydrogel is initially in equilibrium with unsaturated water vapor. What will happen if we isolate the hydrogel from the vapor by immersing the hydrogel in a hydrophobic liquid (i.e., oil)? Upon introducing a cavity on the surface of the hydrogel, the interface energy may suck water out of the hydrogel to form a pure liquid phase (Fig. 1a). On the other hand, osmosis promotes the low-humidity hydrogel to imbibe water. When capillarity balances osmosis, the two phases-hydrogel and pure liquid water-coexist in equilibrium. We call this phenomenon osmocapillary phase separation.
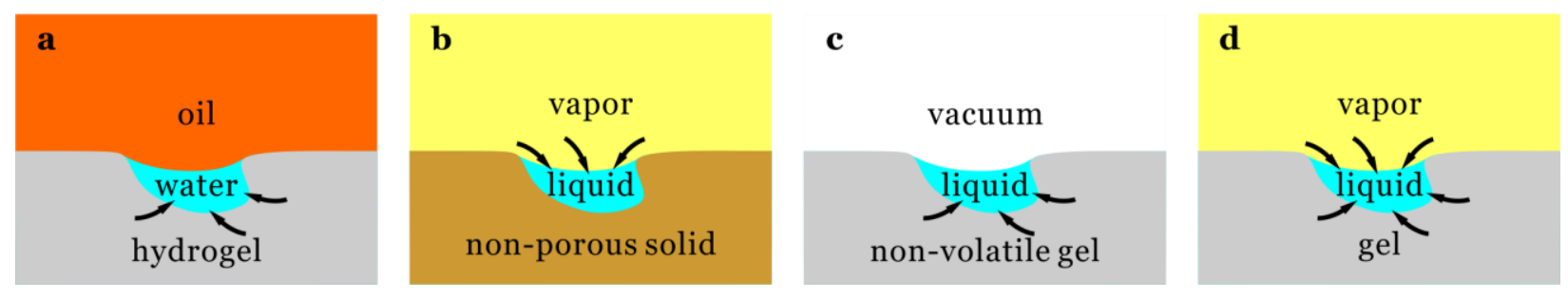

Fig. 1. Formation of a liquid phase in a cavity on an interface. (a) Osmocapillary phase separation. In a cavity on a hydrogel-oil interface, the interface energy competes against osmosis and sucks water out of the hydrogel. (b) Capillary condensation. In a cavity on the interface between a non-porous solid and a vapor, the interface energy causes vapor to condense into liquid water. (c) In a cavity on the surface of a non-volatile gel, osmocapillary phase separation still happens, but capillary condensation is impossible. (d) In a cavity on a gel-vapor interface, osmocapillary phase separation and capillary condensation are concurrent processes.

In the configuration of Fig. 1a, the cavity is filled with liquid water diffusing from the hydrogel, which serves as a reservoir of water. Even without the hydrogel, a cavity on a solid surface may be filled with liquid water condensing from humid air, which is also a reservoir of water (Fig. 1b). Such capillary condensation has been widely studied [12]. Although both osmocapillary phase separation and capillary condensation can fill a cavity on an interface, 
water comes from different reservoirs. In capillary condensation, water comes from the humid air. In osmocapillary phase separation, water comes from the hydrogel.

A polymer network may also imbibe solvent other than water. When a gel of a nonvolatile solvent is exposed to air, capillary condensation is impossible, but osmocapillary phase separation can still happen (Fig.1c). If the solvent of the gel is volatile, capillary condensation and osmocapillary phase separation are concurrent processes (Fig.1d). We show that osmocapillary phase separation dominates over capillary condensation for small cavities.

Because the chemical potential of solvent in a gel can be readily tuned, osmocapillary phase separation can occur in cavities of a large range of sizes. The competition between osmosis and capillarity defines a length, which we call the osmocapillary length. When the osmocapillary length is large compared to the size of the cavity, the capillary force sucks pure liquid solvent out of the gel. We will describe various ways to increase the osmocapillary length. Although osmocapillary phase separation has not been well-studied, related phenomena are important in many applications. Examples include the wettability of gels, the transparency of gels, the liquid bridge at the tip of an atomic force microscope (AFM), the adhesion between a gel and another substance, the surface morphology of gels, and the production of tight oil. We will describe these phenomena at the end of the paper.

\section{Chemical potential of solvent in various states}

We review the chemical potentials of solvent in various states of aggregation. The chemical potential of a pure substance is a function of pressure and temperature, and coincides with the Gibbs function per molecule of the substance. Under the isothermal conditions, the chemical potential $\mu$ of a pure solvent obeys the thermodynamic relation $d \mu=v d P$, where $v$ is the volume of solvent (either vapor or liquid) divided by the number of solvent molecules, and $P$ is the pressure. In thermodynamic equilibrium, the chemical potential of solvent is the same everywhere. We set the chemical potential of solvent to zero when pure liquid solvent and pure 
gaseous solvent coexist in equilibrium. In this reference state, the pressure is the same in the pure liquid and in the pure vapor; we denote the pressure of saturation by $P_{\text {sat. }}$.

When gaseous solvent is isolated from liquid solvent, the pressure in the vapor, $P$, can differ from the pressure of saturation. The vapor is taken to obey the law of ideal gases, $P v=k T$, where $k T$ is the temperature in the unit of energy. An integration of $d \mu=v d P$ gives the chemical potential of solvent in its pure vapor phase:

$$
\mu_{v}=k T \log \left(P / P_{\mathrm{sat}}\right) .
$$

The chemical potential of solvent in the vapor relates to $P / P_{\text {sat. }}$ For a vapor of water, the ratio $P / P_{\text {sat }}$ defines the relative humidity. Equation (1) recovers the reference state when the vapor is saturated-that is, $\mu_{v}=\mathrm{O}$ when $P=P_{\text {sat }}$.

When the pure liquid solvent is isolated from its vapor, the hydrostatic stress in the liquid, $\sigma$, can differ from the pressure of saturation. We take $\sigma$ to be positive when the stress in the liquid is tensile. Liquid solvent is assumed to be incompressible-that is, the volume per solvent molecule in the liquid phase, $\Omega$, is independent of the stress. An integration of $d \mu=v d P$ gives the chemical potential of solvent in its pure liquid phase:

$$
\mu_{l}=-\Omega\left(\sigma+P_{\mathrm{sat}}\right) \text {. }
$$

The chemical potential of solvent in its pure liquid phase is linear in the applied hydrostatic stress. Equation (2) recovers the reference state when the liquid solvent equilibrates with the gaseous solvent-that is, $\mu_{l}=\mathrm{O}$ when $\sigma=P_{\text {sat. }}$.

Experiments are often conducted under the atmospheric pressure, $P_{\mathrm{atm}}$. The air contains the vapor of the solvent, and is modeled as an ideal-gas mixture. The chemical potential of solvent in the air is still given by (1), with $P$ being the partial pressure of solvent in the air, and $P_{\text {sat }}$ still being the pressure of saturation when pure liquid solvent and pure gaseous solvent equilibrate. When the pure liquid is under atmospheric pressure, $\sigma=P_{\mathrm{atm}}$, the chemical potential of solvent in the liquid is $\mu_{l}=\Omega\left(P_{\mathrm{atm}}-P_{\mathrm{sat}}\right)$. For example, $P_{\mathrm{sat}} \sim 3 \mathrm{kPa}$ for water at 
room temperature and $P_{\mathrm{atm}} \sim 100 \mathrm{kPa}$. Consequently, the atmospheric pressure greatly increases the chemical potential of water in its pure liquid phase. Equating the chemical potential of solvent in the liquid and that in the air, we obtain the partial pressure of the solvent in the air in equilibrium with the liquid water:

$$
P_{\mathrm{o}}=P_{\mathrm{sat}} \exp \frac{P_{\mathrm{atm}}-P_{\mathrm{sat}}}{k T / \Omega}
$$

For water, taking $\Omega=3.0 \times 10^{-29} \mathrm{~m}^{3}, T=300 \mathrm{~K}$ and $k=1.38 \times 10^{-23} \mathrm{~J} / \mathrm{K}$, we obtain that $k T / \Omega=$ $138 \mathrm{MPa}$, which is much larger than the atmospheric pressure. Consequently, the atmospheric pressure increases the equilibrium partial pressure of water negligibly, $P_{\mathrm{o}} \sim P_{\text {sat }}$.

Denote the chemical potential of pure liquid solvent under atmospheric pressure by $\mu_{\mathrm{o}}=\Omega\left(P_{\mathrm{atm}}-P_{\mathrm{sat}}\right)$. Rewriting (1), we express the chemical potential of solvent in an ideal-gas mixture as

$$
\mu_{v}=\mu_{\mathrm{o}}+k T \log \left(P / P_{\mathrm{o}}\right)
$$

For pure liquid solvent subject to hydrostatic stress $\sigma$, define an excess stress by $\sigma^{\prime}=\sigma-\left(P_{a t m}-P_{s a t}\right)$. Rewriting (2), we express the chemical potential of solvent in the pure liquid as

$$
\mu_{l}=\mu_{\mathrm{o}}-\Omega \sigma^{\prime}
$$

Now consider a pure liquid solvent with a curved surface. The ambient is subject to the atmospheric pressure. The chemical potential of solvent beneath the curved liquid-vapor interface is

$$
\mu_{\gamma}=\mu_{\mathrm{o}}-\Omega \kappa \gamma
$$

Here $\gamma$ is the energy per unit area of the interface, and $\kappa$ is the sum of the two principal curvatures of the interface. A positive $\kappa$ corresponds to a cavity, and a negative $\kappa$ corresponds to a droplet. Equation (6) also applies to an oil-water interface, where $\gamma$ is the energy per unit area of the oil-water interface, the oil is subject to the atmospheric pressure, and $\mu_{\gamma}$ is the chemical 
potential of water near the curved water-oil interface.

As mentioned before, the chemical potential of solvent in a gel, $\mu_{\text {gel }}$, can be readily changed by equilibrating the gel with a solvent vapor of known partial pressure [11]. The chemical potential of solvent is negative in a gel in equilibrium with an unsaturated solvent vapor. We define the osmotic pressure $\Pi$ of the gel by the relation

$$
\mu_{\mathrm{gel}}=\mu_{\mathrm{o}}-\Omega \Pi
$$

This definition of the osmotic pressure in a gel is analogous to that of the osmotic pressure in a liquid solution. For a liquid solution in equilibrium with a pure solvent, the osmotic pressure in the solution is measurable as a pressure, e.g., by the rise of the solution against gravity. For a gel in equilibrium with a pure liquid solvent, $\mu_{\text {gel }}=\mu_{l}$, the osmotic pressure in the gel balances the excess stress in the pure liquid solvent, $\Pi=\sigma^{\prime}$.

\section{Osmocapillary length}

We now return to the cavity on the hydrogel-oil interface (Fig. 1a). For a hydrogel containing a large quantity of water, the energy per unit area of the hydrogel-oil interface is nearly identical to that of the water-oil interface. When the hydrogel extrudes water, the resulting water-oil interface is less curved than the hydrogel-oil interface, so that the net interface energy reduces. Thus, the capillarity promotes the separation of the hydrogel into two phases: hydrogel and pure liquid water. The water-oil interface is still slightly curved, and sets up a difference in stress on the two sides of the interface, known as the Laplace stress. The oil is under the atmospheric pressure. Inside the cavity, the energy of the oil-water interface causes a state of tensile excess stress in the liquid water $\left(\sigma^{\prime}>0\right)$. Equating the chemical potentials of water, $\mu_{\gamma}=\mu_{l}$, one obtains $\sigma^{\prime}=\gamma \kappa$.

The Laplace stress in the liquid water relative to the oil is tensile, and tends to suck water

out of the hydrogel. On the other hand, osmosis promotes the low-humidity hydrogel to imbibe 
water. When capillarity balances osmosis, the two phases-hydrogel and pure liquid watercoexist in equilibrium. Equating the chemical potentials, $\mu_{\mathrm{gel}}=\mu_{\gamma}$, we obtain the condition of osmocapillary equilibrium:

$$
\Pi=\gamma \kappa .
$$

When the hydrogel is a reservoir of water with a fixed chemical potential (and therefore a fixed osmotic pressure), The condition of equilibrium determines the curvature of the water-oil interface.

The ratio $\gamma / \Pi$ defines a length, which we call the osmocapillary length. If the size of the cavity, $L$, is small comparing to the osmocapillary length, there exists a continuous surface of curvature $\kappa$ covering the cavity that satisfies $\gamma \kappa=\Pi$, and phase separation happens. However, for a large cavity, $L>\gamma / \Pi$, phase separation does not happen. In the limit that the hydrogel is in equilibrium with the saturated vapor, $\Pi=0$, osmocapillary phase separation can occur in a cavity of any size. In the other limit that the hydrogel is completely dry, $\Pi=\infty$, osmocapillary phase separation cannot happen in a cavity of any size.

For a hydrogel prepared in equilibrium with a vapor of relative humidity $P / P_{\mathrm{o}}$, the osmocapillary length is

$$
\frac{\gamma}{\Pi}=-\frac{\gamma \Omega}{k T \log \left(P / P_{\mathrm{o}}\right)}
$$

For a representative values $\quad=70 \mathrm{~mJ} / \mathrm{m}^{2}$ and $k T / \Omega=138 \mathrm{MPa}$, we plot the osmocapillary length as a function of relative humidity (Fig.2). The osmocapillary length decreases steeply at the dry end and increases steeply at the wet end. The osmocapillary length is roughly $5 \mathrm{~nm}$ at $90 \%$ relative humidity, and increases to roughly 50nm at $99 \%$ relative humidity. 


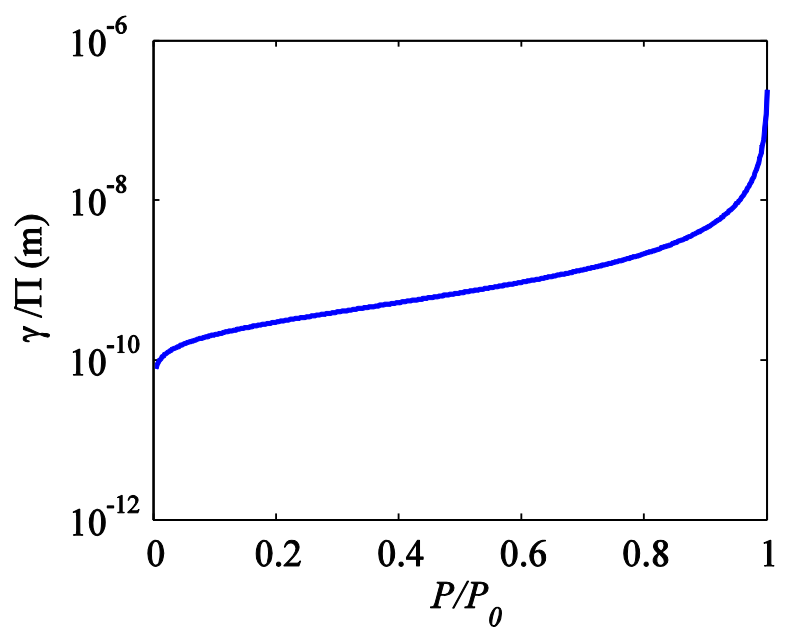

Fig.2. Osmocapillary length for hydrogel prepared at various values of relative humidity.

In a sealed environment, the relative humidity in the vapor can be controlled by an aqueous solution. Let $c$ be the concentration of the solute (i.e., the number of particles of the solute divided by the volume of the solution). The osmotic pressure of the solution is given by the Van't Hoff equation $\Pi=c k T$. This osmotic pressure sets the osmotic pressure in the hydrogel if the vapor over the aqueous solution equilibrates with the hydrogel. In this case, the osmocapillary length is given by

$$
\frac{\gamma}{\Pi}=\frac{\gamma}{c k T}
$$

For example, take the surface tension of an aqueous solution to be still $=70 \mathrm{~mJ} / \mathrm{m}^{2}$. For a sucrose solution of $0.1 \mathrm{M}$, the osmotic pressure is $\Pi=0.25 \mathrm{MPa}$, and the osmocapillary length is $0.28 \mu \mathrm{m}$. For a colloidal solution of $0.1 \mathrm{mM}$, the osmotic pressure will be lowered to $\Pi=0.25 \mathrm{kPa}$, and the osmocapillary length will be increased to $280 \mu \mathrm{m}$.

We now consider a gel of nonvolatile solvent (Fig. 1c). Still, a pure liquid solvent in a cavity equilibrates with solvent in the gel when capillarity balances osmosis, $\gamma \kappa=\Pi$, where $\gamma$ is the energy per unit area of the solvent-air interface, $\kappa$ is the curvature of the solvent-air interface and $\Pi$ is the osmotic pressure in the gel. To observe osmocapillary phase separation, we need 
to reduce the magnitude of the osmotic pressure in the gel-that is, to bring the chemical potential of solvent in the gel to be sufficiently close to that in pure liquid solvent. An effective way to do so is to use a solvent of giant molecules.

Consider a network of polymer swollen with the liquid of the polymer of the same chemical structure. The enthalpy of mixing vanishes, and the mixing of the solvent and network is entirely driven by entropy. Let $V$ be the volume of the networked polymer, $N$ be the number of uncrosslinked polymer chains, and $\Omega$ be the volume per chain. When the uncrosslinked chains form a pure liquid, the volume of the liquid is $N \Omega$, and the number of configurations scales as $W_{\mathrm{o}} \propto(N \Omega)^{N}$. When the uncrosslinked chains and the network form a gel, the volume of the gel is $V+N \Omega$, and the number of configurations scales as $W_{\text {gel }} \propto(V+N \Omega)^{N}$. Consequently, the entropy of mixing is $S_{\mathrm{gel}}-S_{\mathrm{o}}=k \log \left(W / W_{\mathrm{o}}\right)$. The change in the chemical potential of solvent between the two states of aggregation obeys the thermodynamic relation

$$
\begin{aligned}
& \mu_{\mathrm{gel}}-\mu_{\mathrm{o}}=-T \partial\left(S_{\mathrm{gel}}-S_{\mathrm{o}}\right) / \partial N \text {, giving } \\
& \qquad \mu_{\mathrm{gel}}=\mu_{\mathrm{o}}+k T\left(\log \frac{N \Omega}{V+N \Omega}+\frac{V}{V+N \Omega}\right) .
\end{aligned}
$$

Equation (11) corresponds to the entropy of mixing in the Flory-Huggins model [13, 14]; here we have neglected the change in the entropy due to the elasticity of the network. For a gel in which the volume of solvent is comparable to that of the networked polymer, the term in the parenthesis in (11) is of order unity, so that the osmotic pressure is on the order of $\Pi \sim k T / \Omega$. For example, the density of a polymer is on the order of $10^{3} \mathrm{~kg} / \mathrm{m}^{3}$; for a polymer chain of molecular weight of $100,000 \mathrm{~g} / \mathrm{mol}$, the volume of the polymer is about $2 \times 10^{-25} \mathrm{~m}^{3}$ and the osmotic pressure is about $25 \mathrm{kPa}$. The osmotic pressure is greatly reduced for a gel with solvent of long-chain polymers. Taking a representative value of surface energy, $\gamma=20 \mathrm{mN} / \mathrm{m}$, we obtain an osmocapillary length on the order of microns. 


\section{Phase separation along a three-phase contact line}

In a recent experiment, Jensen et al [15] observed the formation of a liquid phase along the contact line of three phases (air, a gel and a solid). In their experiment, spherical glass particle is dropped onto loosely crosslinked PDMS. The PDMS is so soft that it partially engulfs the glass particle. In the loosely crosslinked PDMS, some of the PDMS molecules are not crosslinked, and can migrate out as liquid PDMS. The experiment shows that uncrosslinked PDMS molecules extrude out from the gel and form a liquid phase near the contact line (Fig.3).

We now interpret this experimental observation in terms of osmocapillary phase separation. Liquid PDMS is nonvolatile and the possibility of condensation from vapor is excluded. The three-phase contact line has a singular curvature, which allows osmocapillary phase separation with at any finite $\Pi$. In the experiment, the PDMS network and the PDMS liquid consist of the identical molecular structure except for a tiny fraction of crosslinkers. Consequently, enthalpy of mixing vanishes. The reported molecular weight of the precrosslinked PDMS is $28000 \mathrm{~g} / \mathrm{mol}$, and the density of PDMS is $0.97 \mathrm{~kg} / \mathrm{m}^{3}$ [16]. The volume occupied by each polymer chain can be estimated based on the molar weight of the polymer, which gives $\Omega=4.8 \times 10^{-26} \mathrm{~m}^{3}$. Assuming the volume fraction of the uncrosslinked PDMS is high, say 90\%, Equation (12) gives the osmotic pressure $\Pi \sim 9.1 \mathrm{kPa}$. Taking the surface energy of the PDMS as $20 \mathrm{mN} / \mathrm{m}$. The corresponding osmocapillary length is $\gamma / \Pi=2.2 \mu \mathrm{m}$. This estimate is comparable to the size of the liquid phase observed in the experiment. A full analysis of the experiment requires a theory that couples osmosis, capillarity and elasticity, which is beyond the scope of this paper. 


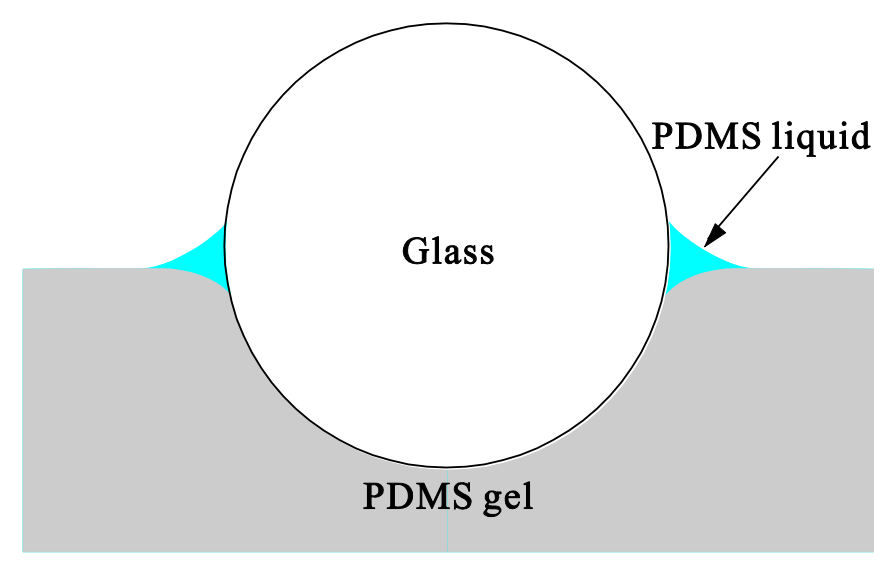

Fig.3. When a glass particle is dropped on a soft PDMS gel swollen with liquid PDMS, liquid PDMS extrudes out near the three-phase contact line.

\section{Osmocapillary phase separation vs. capillary condensation}

When the surface of a solid is exposed to the ambient air, liquid water may condense out from humid air near small cavities on a solid surface (Fig. 1b). This capillary condensation has important consequence for micro-electro-mechanical systems [17-19], self-assembly [20-22], and atomic force microscopes [23-25].

We now consider a polymer network swollen with a solvent and exposed to air (Fig. 1d). The solvent is volatile, and a vapor of the solvent exists in the air. Consequently, capillary condensation and osmocapillary phase separation are concurrent processes. Thermodynamic equilibrium requires that the chemical potential of solvent be the same everywhere, $\mu_{\mathrm{gel}}=\mu_{l}=\mu_{\gamma}=\mu_{v}$, giving that

$$
\Pi=\sigma=\gamma \kappa=-\frac{k T}{\Omega} \log \left(\frac{P}{P_{\text {sat }}}\right) .
$$

The last equation is the Kelvin relation commonly used to analyze capillary condensation.

Equation (12) shows that the condition of equilibrium for osmocapillary phase separation is identical to that for capillary condensation. However, the kinetics of the two 
processes are different. Capillary condensation is due to the condensation of solvent from the vapor, whereas osmocapillary phase separation is due to the diffusion of solvent in the gel.

We compare condensation from the vapor and diffusion in the gel using basic kinetic theory [26-28]. The average velocity of solvent molecules in the vapor is $v=\sqrt{3 k T / m}$, where $m$ is the mass per molecule. The law of ideal gases gives the number of solvent molecules per unit volume of vapor, $c=P_{o} / k T$, where $P_{o}$ is the equilibrium partial pressure of solvent in the vapor. The rate of condensation scales as $d n / d t \sim c v L^{2}$, where $n$ is the number of solvent in the liquid phase, and $L$ is the length scale of the liquid phase. Note that $n \sim L^{3} / \Omega$.

Consequently, the relaxation time for capillary condensation scales as $\tau_{\text {cond }} \sim L \sqrt{m k T} / P_{0} \Omega$. On the other hand, the relaxation time for osmocapillary phase separation scales as $\tau_{\text {osmo }} \sim L^{2} / D$, where $D$ is the diffusivity of the solvent in the gel.

Equating the relaxation times of the two kinetic processes, $\tau_{\text {cond }}=\tau_{\text {osmo }}$, we obtain a length scale $L_{\text {cross }}=D \sqrt{m k T} / P_{0} \Omega$. Take orders of magnitude representative of water, $m \sim 10^{-}$ ${ }^{26} \mathrm{~kg}, \Omega \sim 10^{-29} \mathrm{~m}^{3}, k T \sim 1 \mathrm{O}^{-21} \mathrm{~J}$ and $\mathrm{P}_{\mathrm{o}} \sim 1 \mathrm{kPa}$. The diffusivity of water in hydrogel is roughly $\mathrm{D} \sim$ $10^{-9} \mathrm{~m}^{2} / \mathrm{s}$ [29]. The length scale is estimated as $L_{\text {cross }}=10^{-7} \mathrm{~m}$. This means that, for hydrogels, at sub-micron scale the osmocapillary phase separation dominates over capillary condensation. In the limit of a gel with a nonvolatile solvent, the crossover length approaches infinity, and the formation of the liquid phase is due entirely to osmocapillary phase separation.

We have identified two length scales: the osmocapillary length $\gamma / \Pi$, and the kinetic crossover length $L_{\text {cross }}=D \sqrt{m k T} / P_{0} \Omega$. Experimental observations of a cavity on a gel-air interface will depend on how the size of the cavity $L$ compares with these two length scales (Fig.4). When osmosis prevails over capillarity, no phase separation occurs. When capillarity 
prevails over osmosis, phase separation occurs, either by the diffusion of solvent in the gel, or be the condensation of solvent from the vapor.

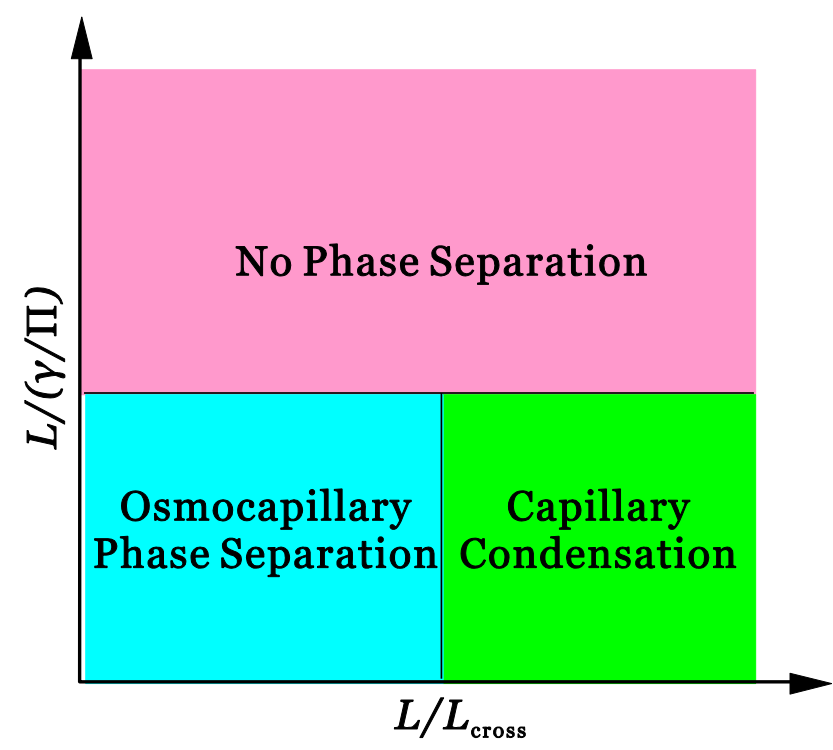

Fig.4. Osmocapillary phase separation occurs when the size of the cavity $L$ is small compared to two lengths,, the osmocapillary lengh $\gamma / \Pi$, and the kinetic crossover length $L_{\text {cross }}=D \sqrt{m k T} / P_{\mathrm{o}} \Omega$.

\section{Osmocapillary phase separation in phenomena of practical significance}

Like capillary condensation, osmocapillary phase separation is expected to be important in many applications. Here we briefly discuss several examples. Osmocapillary phase separation may change the wettability of the gel surface (Fig.5a). The gel and its solvent can have different surface property if the gel has a low swelling ratio, or if the gel carries some functional particles like clay [30]. A droplet of liquid immiscible with the solvent may have different wettability towards the solvent and towards the gel. When the osmotic pressure inside the gel is low, osmocapillary phase separation covers most surface asperities with the solvent, and the wettability of the surface is close to that of the pure solvent. When the osmotic pressure inside the gel is high, osmocapillary phase separation only covers a tiny portion of the surface asperities, and the wettability of the surface is close to that of the gel. 
Osmocapillary phase separation may also change the optical property of the gel (Fig.5b). Depending on the fabrication process, the surface of a gel can be rough and scatter light, resulting in a translucent material. Usually, the difference in refractive index between the gel and the solvent is much smaller than the difference between the gel and the air. With low osmotic pressure, osmocapillary phase separation covers all the asperities, and the material is transparent. With high osmotic pressure, the rough surface scatters light, and the material is translucent.

The examples of Fig.5a and Fig.5b show that osmocapillary phase separation enables switching of the surface property by tuning the osmotic pressure, which could be done by connecting the gel with a reservoir of solvent or applying stress. The same principle has been demonstrated in the "self-healing, slippery liquid infused porous surface" (SLIPS) [31, 32]. In that case, lubricant is infused into a porous material, and the wettability of the porous material is tuned by changing the pore pressure of the lubricant. If we accept pore pressure as an extension of osmotic pressure, these experiments fit into the theory of osmocapillary phase separation.

Osmocapillary phase separation may result in a liquid bridge at the tip of an atomic force microscope (Fig.5c). As discussed earlier, below the kinetic crossover length, osmocapillary phase separation dominates over capillary condensation. Consequently, the liquid bridge draws liquid from the gel, rather than from the ambient air. The difference in kinetics can result in noticeable difference if the atomic force microscope works in the dynamic modes [33]. In addition, capillary condensation may be avoided by immersing the sample in a cover liquid. If the cover liquid is immiscible with the solvent, osmocapillary phase separation can still result in a liquid bridge. For a gel with nonvolatile solvent, test in vacuum can also avoid capillary condensation, but osmocapillary phase separation can still result in liquid bridge as well.

As demonstrated by Jensen et al [15], osmocapillary phase separation produce a liquid ring along the three-phase contact line (Fig.5d). It is known that liquid phase around contact 
line can strongly influence adhesion [34]. This implies that the osmocapillary phase separation will also affect adhesion.

When a soft material is compressed beyond a critical level, the surface of the material forms creases $[35,36]$. Creases account for many naturally observed phenomena, including the creases on our skin and the sulci in the brain. If a crease happens on the surface of a gel, the selfcontact leads to a singularity in the curvature of the gel-air interface. Such a singularity may cause osmocapillary phase separation (Fig.5e), although we are unaware of any experimental observations.

Singularity in the curvature may also happen at the tip of a crack, where osmocapillary phase separation is expected (Fig.5f). Depending on the size of the crack opening relative to the osmocapillary length, the solvent may propagate a long distance along the crack. If we think porous rock as a special case of gel and think crude oil as its solvent, such a phenomenon has been widely used in the production of tight oil through hydraulic fracture [37].

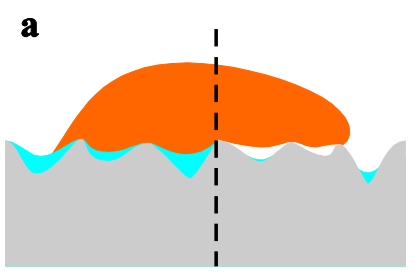

Wettability

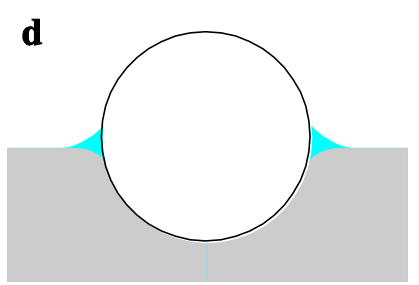

Adhesion

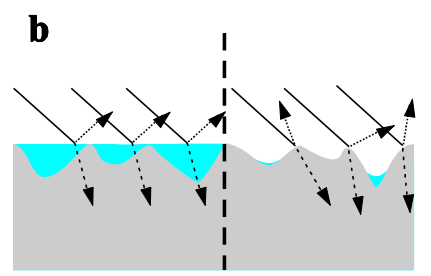

Optical property

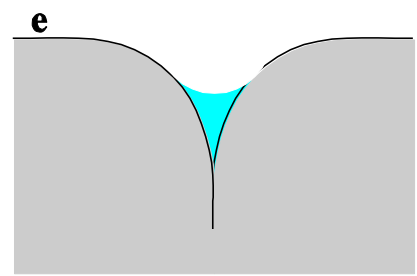

Crease

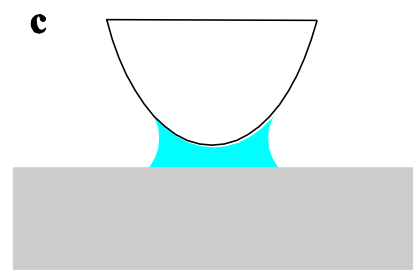

AFM

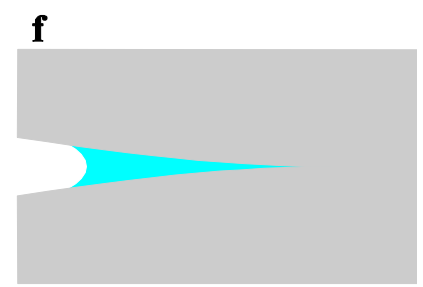

Crack

Fig.5. Osmocapillary phase separation may relate to a variety of phenomena. (a) Osmocapillary phase separation may change the wettability between a gel and an immiscible liquid. (b) Osmocapillary phase separation may switch a translucent material to a transparent material by covering the surface roughness. (c) Osmocapillary phase separation may result in a liquid bridge 
at the tip of an atomic force microscope. (d) Osmocapillary phase separation changes the adhesion between two materials. (d) Osmocapillary phase separation may happen near the tip of a crease. (e) Osmocapillary phase separation may happen near the tip of a crack, extracting liquid out along the crack.

\section{Conclusion}

For a pure liquid solvent in a cavity to coexist in equilibrium with a gel, the Laplace tension in the pure liquid solvent must equal the osmotic pressure in the gel. The competition between capillarity and osmosis defines the osmocapillary length. A cavity smaller than the osmocapillary length draws solvent from the gel and the vapor concurrently. Diffusion of the solvent in the gel prevails over the condensation of the solvent from the vapor when the cavity is smaller than a kinetic crossover length. Osmocapillary phase separation can still occur even when the gel is isolated from vapor phase, or when the solvent of the gel is nonvolatile. Osmocapillary phase separation may be important in many applications, including the wettability of gels, the transparency of gels, the liquid bridge at the tip of an atomic force microscope, the adhesion on gel, the surface morphology of gels, and the production of tight oil.

Acknowledgements. This work is supported by MRSEC (DMR-0820484).

\section{Reference}

1. Hammel, H.T. and P.F. Scholander, Osmosis and tensile solvent. 2012: Springer Science \& Business Media.

2. Helfferich, F.G., Ion exchange. 1962: Courier Corporation.

3. Holbrook, N.M. and M.A. Zwieniecki, Transporting water to the tops of trees. Physics Today, 2008. 61(1): p. 76.

4. Tyree, M.T. and M.H. Zimmermann, Xylem structure and the ascent of sap. 2013: Springer Science \& Business Media.

5. Wheeler, T.D. and A.D. Stroock, The transpiration of water at negative pressures in a synthetic tree. Nature, 2008. 455(7210): p. 208-12. 
6. Beebe, D.J., J.S. Moore, J.M. Bauer, Q. Yu, R.H. Liu, C. Devadoss, and B.-H. Jo, Functional hydrogel structures for autonomous flow control inside microfluidic channels. Nature, 2000. 404(6778): p. 588-590.

7. Zwieniecki, M.A., P.J. Melcher, and N.M. Michele Holbrook, Hydrogel control of xylem hydraulic resistance in plants. Science, 2001. 291(5506): p. 1059-62.

8. Masuda, F. Trends in the development of superabsorbent polymers for diapers. in ACS symposium series. 1994.

9. Cai, S., Y. Lou, P. Ganguly, A. Robisson, and Z. Suo, Force generated by a swelling elastomer subject to constraint. Journal of Applied Physics, 2010. 107(10): p. 103535.

10. Kucera, J., Reverse Osmosis: Industrial Processes and Applications. 2015: John Wiley \& Sons.

11. Illeperuma, W.R.K., J.-Y. Sun, Z. Suo, and J.J. Vlassak, Force and stroke of a hydrogel actuator. Soft Matter, 2013. 9(35): p. 8504.

12. Hunter, R.J., Foundations of colloid science. 2001: Oxford University Press.

13. Huggins, M.L., Solutions of long chain compounds. The Journal of Chemical Physics, 1941. 9(5): p. 440-440.

14. Flory, P.J., Thermodynamics of High Polymer Solutions. The Journal of Chemical Physics, 1942. 10(1): p. 51.

15. Jensen, K.E., R. Sarfati, R.W. Style, R. Boltyanskiy, A. Chakrabarti, M.K. Chaudhury, and E.R. Dufresne, Wetting and phase separation in soft adhesion. Proceedings of the National Academy of Sciences, 2015. 112(47): p. 14490-14494.

16. Number obtained from the supplier's website. http://shop.gelest.com/Product.aspx?catnum=DMS-V31\&Index=0\&TotalCount=2

17. Maboudian, R., Critical Review: Adhesion in surface micromechanical structures. Journal of Vacuum Science \& Technology B: Microelectronics and Nanometer Structures, 1997. 15(1): p. 1.

18. Van Spengen, W.M., R. Puers, and I. De Wolf, On the physics of stiction and its impact on the reliability of microstructures. Journal of Adhesion Science and Technology, 2003. 17(4): p. 563-582.

19. Yapu, Z., Stiction and anti-stiction in MEMS and NEMS. Acta Mechanica Sinica, 2003. 19(1): p. 1-10.

20. Gracias, D.H., J. Tien, T.L. Breen, C. Hsu, and G.M. Whitesides, Forming electrical networks in three dimensions by self-assembly. Science, 2000. 289(5482): p. 1170-1172.

21. Srinivasan, U., D. Liepmann, and R.T. Howe, Microstructure to substrate self-assembly using capillary forces. Microelectromechanical Systems, Journal of, 2001. 10(1): p. 1724.

22. Pokroy, B., S.H. Kang, L. Mahadevan, and J. Aizenberg, Self-organization of a mesoscale bristle into ordered, hierarchical helical assemblies. Science, 2009. 323(5911): p. 237240.

23. Riedo, E., F. Levy, and H. Brune, Kinetics of capillary condensation in nanoscopic sliding friction. Phys Rev Lett, 2002. 88(18): p. 185505.

24. Sahagún, E., P. García-Mochales, G.M. Sacha, and J.J. Sáenz, Energy Dissipation due to Capillary Interactions: Hydrophobicity Maps in Force Microscopy. Physical Review Letters, 2007. 98(17).

25. Chen, S.H. and A.K. Soh, The capillary force in micro- and nano-indentation with different indenter shapes. International Journal of Solids and Structures, 2008. 45(10): p. 3122-3137.

26. Mullins, W.W., Theory of thermal grooving. Journal of Applied Physics, 1957. 28(3): p. 333-339.

27. McDonald, J.E., Homogeneous nucleation of vapor condensation. II. Kinetic aspects. Am. J. Phys, 1963. 31(1): p. 31-41. 
28. Mullins, W.W., Flattening of a Nearly Plane Solid Surface due to Capillarity. Journal of Applied Physics, 1959. 30(1): p. 77.

29. Cai, S., Y. Hu, X. Zhao, and Z. Suo, Poroelasticity of a covalently crosslinked alginate hydrogel under compression. Journal of Applied Physics, 2010. 108(11): p. 113514.

30. Xiang, Y., Z. Peng, and D. Chen, A new polymer/clay nano-composite hydrogel with improved response rate and tensile mechanical properties. European Polymer Journal, 2006. 42(9): p. 2125-2132.

31. Wong, T.S., S.H. Kang, S.K. Tang, E.J. Smythe, B.D. Hatton, A. Grinthal, and J. Aizenberg, Bioinspired self-repairing slippery surfaces with pressure-stable omniphobicity. Nature, 2011. 477(7365): p. 443-7.

32. Yao, X., Y. Hu, A. Grinthal, T.S. Wong, L. Mahadevan, and J. Aizenberg, Adaptive fluidinfused porous films with tunable transparency and wettability. Nat Mater, 2013. 12(6): p. 529-34.

33. Garcia, R. and R. Perez, Dynamic atomic force microscopy methods. Surface science reports, 2002. 47(6): p. 197-301.

34. Maugis, D. and B. Gauthier-Manuel, JKR-DMT transition in the presence of a liquid meniscus. Journal of Adhesion Science and Technology, 1994. 8(11): p. 1311-1322.

35. Hong, W., X. Zhao, and Z. Suo, Formation of creases on the surfaces of elastomers and gels. Applied Physics Letters, 2009. 95(11): p. 111901.

36. Hohlfeld, E. and L. Mahadevan, Unfolding the sulcus. Phys Rev Lett, 2011. 106(10): p. 105702.

37. Clarkson, C.R. and P.K. Pedersen. Tight oil production analysis: adaptation of existing rate-transient analysis techniques. in Canadian Unconventional Resources and International Petroleum Conference. 2010. Society of Petroleum Engineers. 